\title{
Thalamic Iron Differentiates Primary-Progressive and Relapsing-Remitting Multiple Sclerosis
}

\author{
(D) A. Burgetova, (DP. Dusek, (D) M. Vaneckova, (DD. Horakova, (D). Langkammer, (D). Krasensky, (D). Sobisek, (DP. Matras, \\ (i) M. Masek, and (D) Z. Seidl

\begin{abstract}
BACKGROUND AND PURPOSE: Potential differences between primary progressive and relapsing remitting multiple sclerosis are the subject of ongoing controversial discussions. The aim of this work was to determine whether and how primary-progressive and relapsingremitting multiple sclerosis subtypes differ regarding conventional MR imaging parameters, cerebral iron deposits, and their association with clinical status.
\end{abstract}

MATERIALS AND METHODS: We analyzed 24 patients with primary-progressive MS, 80 with relapsing-remitting MS, and 20 healthy controls with 1.5T MR imaging for assessment of the conventional quantitative parameters: T2 lesion load, T1 lesion load, brain parenchymal fraction, and corpus callosum volume. Quantitative susceptibility mapping was performed to estimate iron concentration in the deep gray matter.

RESULTS: Decreased susceptibility within the thalamus in relapsing-remitting MS compared with primary-progressive MS was the only significant MR imaging difference between these MS subtypes. In the relapsing-remitting MS subgroup, the Expanded Disability Status Scale score was positively associated with conventional parameters reflecting white matter lesions and brain atrophy and with iron in the putamen and caudate nucleus. A positive association with putaminal iron and the Expanded Disability Status Scale score was found in primary-progressive MS

CONCLUSIONS: Susceptibility in the thalamus might provide additional support for the differentiation between primary-progressive and relapsing-remitting MS. That the Expanded Disability Status Scale score was associated with conventional MR imaging parameters and iron concentrations in several deep gray matter regions in relapsing-remitting MS, while only a weak association with putaminal iron was observed in primary-progressive MS suggests different driving forces of disability in these MS subtypes.

ABBREVIATIONS: BPF = brain parenchymal fraction; CCV = corpus callosum volume; CN = caudate nucleus; DGM = deep gray matter; EDSS = Expanded Disability Status Scale; GP = globus pallidus; HC = healthy controls; Put = putamen; QS = quantitative susceptibility; QSM = quantitative susceptibility mapping; PPMS = primary-progressive multiple sclerosis; RRMS = relapsing-remitting multiple sclerosis; RR $_{\mathrm{EM}}=$ relapsing-remitting EDSS-matched group; TILL = T1 lesion load; $\mathrm{T} 2 \mathrm{LL}=\mathrm{T} 2$ lesion load

athologic cerebral iron accumulation in multiple sclerosis is a consistent finding in MR imaging and neuropathologic stud-
Received August 25, 2016; accepted after revision January 26, 2017.

From the Departments of Radiology (A.B., M.V., J.K., P.M., M.M., Z.S.) and Neurology (P.D., D.H.), Center of Clinical Neuroscience, First Faculty of Medicine, Charles University and General University Hospital in Prague, Prague, Czech Republic; Institute of Neuroradiology (P.D.), University Medicine Göttingen, Göttingen, Germany; Department of Neurology (C.L.), Medical University of Graz, Graz, Austria; and Department of Statistics and Probability (L.S.), University of Economics, Prague, Czech Republic.

This study was supported by the grants RVO VFN64165, 15-25602A Czech Ministry of Health, and GA CR 16-03322S. L.S. was supported by the Faculty of Informatics and Statistics, University of Economics, Prague.

Please address correspondence to Andrea Burgetova, MD, Department of Radiology, First Faculty of Medicine, Charles University and General University Hospital in Prague, Katerinska 30, 12808 Prague 2, Czech Republic; e-mail: andrea.burgetova@vfn.cz

- Indicates open access to non-subscribers at www.ajnr.org ies. Abnormal iron deposits were detected particularly in the deep gray matter (DGM) - that is, in the putamen, caudate nucleus $(\mathrm{CN})$, and globus pallidus (GP) with iron-sensitive MR imaging techniques such as R2* relaxometry, magnetic field correlation imaging, phase imaging, and quantitative susceptibility mapping (QSM). ${ }^{1-8}$ Neuropathologic studies in MS confirmed increased iron content in both glial cells and neurons in DGM associated with degenerative changes, ${ }^{9}$ while overall iron loss was observed in normal-appearing white matter. ${ }^{10}$ An increase in iron concentration appears to be an early phenomenon, with the highest amounts of accumulation occurring during the transitions from clinically isolated syndrome to definite MS. ${ }^{11}$

Indicates article with supplemental on-line tables.

Indicates article with supplemental on-line photos.

http://dx.doi.org/10.3174/ajnr.A5166 
Currently, little information is available on the differences in iron content in various MS subtypes. Primary-progressive MS (PPMS) is a subtype characterized by a steady progression without relapses and worse response to immunosuppressant drugs. It has been postulated that underlying mechanisms and measures of disability progression in PPMS may be different from those in relapsing-remitting MS (RRMS). In particular, inflammation may be less prominent compared with neurodegeneration in the PPMS subtype. However, neuroimaging studies supporting this theory are lacking. It remains unclear whether PPMS and RRMS subtypes differ in iron accumulation in the DGM. Differences in demographic and clinical data cause difficulties in the comparison of these 2 subtypes. PPMS affects older age groups than RRMS, with a peak incidence in the fifth and sixth decades; the male-to-female ratio is typically $1: 1$ compared with 1:3 reported in most RRMS trials. Additionally, the Expanded Disability Status Scale (EDSS) score is higher in patients with PPMS than in those with RRMS, with the same disease duration indicating faster disease progression in PPMS. ${ }^{12}$

The primary goal of this study was to compare iron content and conventional MR imaging parameters such as T2 lesion load (T2LL), T1 lesion load (T1LL), brain parenchymal fraction (BPF), and corpus callosum volume (CCV) in RRMS and PPMS. We were particularly interested in whether these MS subtypes differ in iron concentration and whether iron accumulation has a different impact on disability in PPMS compared with RRMS. From several MR imaging-based techniques enabling the assessment of iron concentration in the brain, ${ }^{13}$ we chose quantitative susceptibility mapping (QSM). Postmortem validation studies have demonstrated that QSM in the DGM is tightly correlated with iron concentration. ${ }^{14}$ QSM has been shown to correlate with R2 and R2* relaxometry, but compared with these techniques, it does not require multiecho data, has a higher dynamic range, and might be more sensitive to smaller tissue-susceptibility changes. ${ }^{15-18}$

The secondary goal was to investigate the correlation between quantitative MR imaging parameters and clinical disability measured by the EDSS in both MS subtypes.

\section{MATERIALS AND METHODS Research Subjects}

Twenty-four patients with PPMS, 20 age- and sex-matched healthy controls (HC), and 303 patients with RRMS were investigated. Patients with RRMS and PPMS were diagnosed according to the clinical classification of Lublin et al. ${ }^{19}$ Of the total 28 patients with PPMS from the data base of our MS center, 4 patients were excluded due to contraindications to MR imaging, refusal to participate in this study, moving away, and incomplete set of data, respectively. Patients with RRMS who had routine MR imaging examinations from September 2013 to September 2015 were asked to participate in this study; 303 patients were investigated. From the whole sample of 303 patients with RRMS, 2 groups have been identified by distributional matching to the PPMS group by using the MatchIt Library (Version 2.4-21), ${ }^{20}$ as implemented in the R Statistical and Computing Software (http://www.r-project.org/). The first RRMS group consisting of 80 patients was obtained by matching RRMS to PPMS according to age and sex. The second RRMS group was obtained by an independent procedure of matching RRMS to PPMS according to age, sex, and EDSS score. This additional EDSS matching, performed to control for higher disease severity in the PPMS group, yielded a group of 40 patients, referred to as $\mathrm{RR}_{\mathrm{EDSS}-\text { Matched }}\left(\mathrm{RR}_{\mathrm{EM}}\right)$; RRMS and $\mathrm{RR}_{\mathrm{EM}}$ groups are partially overlapping with 29 patients included in both groups. Seventy-nine percent of patients from the RRMS group, $75 \%$ from the $\mathrm{RR}_{\mathrm{EM}}$ group, and 29\% from the PPMS group were on a long-term MS-specific treatment (On-line Table 1). Written informed consent was obtained from all patients, and the research was approved by the local medical ethics committee at First Faculty of Medicine, Charles University and General University Hospital in Prague, Czech Republic.

\section{MR Imaging Acquisition and Image Processing}

The examinations were performed by using a $1.5 \mathrm{~T}$ MR imaging system (Gyroscan NT; Philips Healthcare, Best, the Netherlands); a standard quadratic head coil was used. The protocol included FLAIR (150 axial sections, TR $=1000 \mathrm{~ms}, \mathrm{TE}=140 \mathrm{~ms}$, TI $=$ $2600 \mathrm{~ms}$, spatial resolution $=1 \times 1 \times 1 \mathrm{~mm}^{3}$, scan duration $=10$ minutes 16 seconds), T1-weighted imaging (fast-field echo/3D, 150 axial sections, $\mathrm{TR}=25 \mathrm{~ms}, \mathrm{TE}=5.01 \mathrm{~ms}$, spatial resolution $=$ $1 \times 1 \times 1 \mathrm{~mm}^{3}$, scan duration $=12$ minutes 48 seconds), and susceptibility-weighted imaging (fast-field echo/3D, 100 axial sections, $\mathrm{TR}=48.1 \mathrm{~ms}, \mathrm{TE}=33.2 \mathrm{~ms}$, spatial resolution $=0.8 \times$ $0.8 \times 2.0 \mathrm{~mm}^{3}$, scan duration $=6$ minutes 30 seconds) pulse sequences.

Automated volumetric image analysis was performed with the in-house-developed ScanView software (http://www.scanview.cz/) as described previously. ${ }^{21-23}$ In brief, after standard image processing, image signal intensity was normalized (peak $=10,000$, $\mathrm{WM}=5000$ artificial units) and the volume of T2 lesions (T2LL) was measured in homogenized and filtered FLAIR images as the area exceeding $140 \%$ of the WM intensity and the size of 11 voxels. Next, T2 hyperintensities were transformed to the T1-weighted image and the volume of hypointensities (T1LL) was calculated as the area with signal below $70 \%$ of the WM. In addition, the number of thalamic lesions was counted by a single rater on FLAIR images. BPF (volume of the brain parenchyma divided by the volume of the brain parenchyma and CSF space) and CCV were measured on the T1-weighted image. For CCV measurement, transversal sections were reconstructed to the sagittal plane. After smoothing and edge-enhancing, we applied filters and the area of the corpus callosum was outlined automatically on 7 sections, including the midsagittal section and 3 adjacent sections to the left and to the right. The resulting areas bounded by the curve were then recorded in all 7 sagittal reconstructions, and average value was calculated. The corpus callosum volume was calculated as mean area multiplied by the total thickness of 7 sections.

The reproducibility of T1LL, T2LL, BPF, and CCV measurements was evaluated by using MR imaging data of 164 patients with MS who were examined every 8 weeks throughout 1 year. Interclass correlation coefficients of 2 measurements performed within 8 weeks were 0.9983 for T1/T2LL, 0.9920 for BPF, and 0.9985 for CCV. The estimated reproducibility error of the automatic WM lesion segmentation algorithm was calculated to be $10 \%$ for small lesions $\left(<1 \mathrm{~cm}^{3}\right)$ and $2 \%-3 \%$ for larger lesions.

QSM images were reconstructed by using a total generalized 


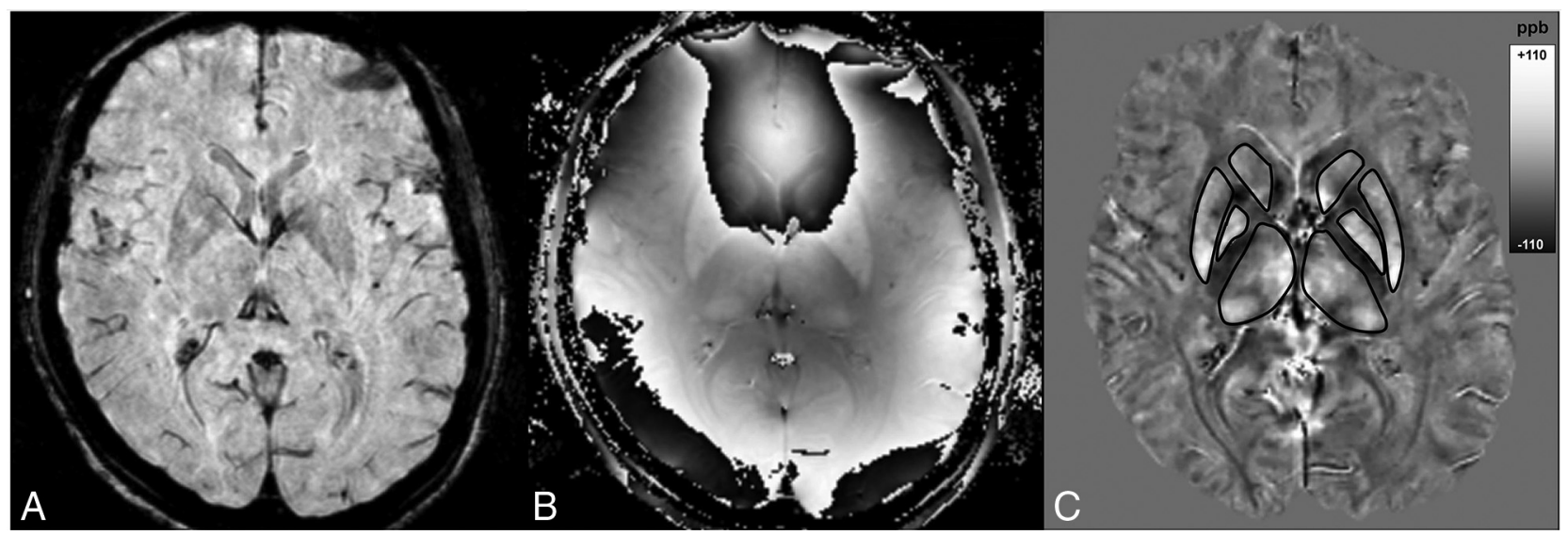

FIG 1. Sample MR image section depicting gradient-echo magnitude $(A)$, raw phase $(B)$, and quantitative susceptibility map $(C)$ with ROIs outlined in the caudate nucleus, putamen, globus pallidus, and thalamus.

variation method as previously described. ${ }^{24}$ Briefly, the reconstruction consisted of Laplacian unwrapping, background field removal, and dipole inversion by using the total generalized variation regularization in a single integrated step. Susceptibility was measured in the following ROIs: CN, putamen (Put), GP, and thalamus. ROIs were determined by using automated segmentation of the T1-weighted image with FreeSurfer, Version 4.5 (http://surfer.nmr.mgh.harvard.edu/). QSM images were rigidly aligned with the T1-weighted images, and subsequently regional median susceptibilities in parts per billion were measured (Fig. 1).

To check the performance of the automatic segmentation algorithm, we compared it with manual segmentation, which was considered ground truth. The extent of spatial overlap between the manual and automatic segmentations was evaluated by using comparative DICE scores. The CN, Put, GP, and thalamus outlines were manually segmented by a single rater in 3 randomly selected healthy subjects by using ITK-SNAP software (www. itksnap.org/). ${ }^{25}$ The $\mathrm{CN}$ and Put were segmented on T1-weighted image; the GP and thalamus were segmented while simultaneously using coregistered T1-weighted and QSM images. The mean comparative DICE scores (1.0 is perfect alignment) were 0.76 (range, $0.71-0.80$ ) for the $\mathrm{CN}, 0.79$ (range, $0.79-0.80$ ) for the Put, 0.74 (range, $0.68-80$ ) for the GP, and 0.79 (range, 0.770.81 ) for the thalamus.

Because thalamic iron distribution is inhomogeneous, we have additionally performed manual segmentation of the entire thalamic outline and pulvinar outline in all subjects with QSM and T1-weighted images. Consequently, susceptibility in parts per billion was measured in the thalamus, pulvinar, and thalamus without the pulvinar.

\section{Statistical Analysis}

We used $t$ tests or nonparametric Mann-Whitney $U$ tests with a nonpooled SD to compare between-group differences in mean or median values of demographic, clinical, and MR imaging characteristics and median susceptibilities. Age was used as a covariate in the analysis to correct for age-related increases in cerebral iron concentration. ${ }^{26}$ The Benjamini-Hochberg procedure with $P<$ .05 was used to minimize the false discovery rate. The relations between EDSS and MR imaging metrics within each group were investigated by the Spearman correlation coefficient. Addition- ally, interaction analysis by using the multivariate general linear model was performed to examine whether the association between conventional MR imaging parameters and susceptibility in DGM nuclei differs by MS subtype. Interaction between MR imaging parameters and EDSS by MS subtype was also tested. Thus, patients were categorized into 1 of 4 classes according to EDSS category and MS subtype (ie, PPMS with EDSS $\leq 4$, PPMS with EDSS $>4$, RRMS with EDSS $\leq 4$, RRMS with EDSS $>4$ ). ANOVA and paired $t$ tests were used to compare differences in means of MR imaging parameters among these subgroups. All analyses were performed with the statistical software R (www. r-project.org/); reported $P$ values are 2-tailed.

\section{RESULTS}

\section{Clinical and Conventional MR Imaging Parameters: Comparison of RRMS, PPMS, and Controls}

MR imaging metrics in RRMS were compared with those in the PPMS and HC groups. The clinical disability (EDSS) distribution of patients with RRMS differs significantly from that in patients with PPMS $(P<.001)$. The PPMS group was, therefore, also compared with the $\mathrm{RR}_{\mathrm{EM}} \mathrm{MS}$ subgroup, consisting of 40 patients matched for EDSS scores. Subject demographic and clinical data and comparison of conventional MR imaging parameters among RRMS, PPMS, and HC are listed in Table 1. The PPMS group showed significantly higher T1LL $(P=.023)$ relative to the RRMS group, while no significant differences were found between PPMS and $\mathrm{RR}_{\mathrm{EM}} \mathrm{MS}$ groups in conventional MR imaging metrics.

\section{Comparison of Susceptibility among MS Subtypes and HC} Compared with HC, both MS groups had significantly increased quantitative susceptibility $(\mathrm{QS})$ values in the putamen (HC versus RRMS, $P=.042$, and PPMS, $P=.009$, respectively) (Fig. 2A). Regional susceptibility was significantly lower in the thalamus in the RRMS group compared with $\mathrm{HC}(P=.004)$. In contrast, there was no such decrease in thalamic susceptibility in the PPMS group compared with HC ( $P=$.757). The only significant difference between MS subtypes found was lower regional susceptibility in the thalamus in the RRMS compared with the PPMS group $(P=.007)$ (Table 2 and Fig. $2 B$ ). Lower QS values in the thalamus were also observed in the $\mathrm{RR}_{\mathrm{EM}}$ group compared with PPMS $(P=.007)$. Thalamic QS values were likely not influenced by demyelinating lesions 
Table 1: Demographic and clinical data and conventional MRI metrics in HC, RRMS, RR EM $_{\text {, and PPMS groups }}{ }^{\mathrm{a}}$

\begin{tabular}{|c|c|c|c|c|c|c|c|}
\hline & \multirow[b]{2}{*}{$\begin{array}{l}\text { HC 20 } \\
(8 / 12)\end{array}$} & \multirow[b]{2}{*}{$\begin{array}{c}\text { RRMS } 80 \\
(32 / 48)\end{array}$} & \multirow[b]{2}{*}{$\begin{array}{c}\mathrm{RR}_{E M} \mathrm{MS} 40 \\
(16 / 24)\end{array}$} & \multirow[b]{2}{*}{$\begin{array}{c}\text { PPMS } 24 \\
(9 / 15)\end{array}$} & \multicolumn{3}{|c|}{$P$ Value $^{\mathrm{b}}$} \\
\hline & & & & & $\begin{array}{c}\text { PPMS vs RRMS } \\
\left(R_{E_{E M}}\right)\end{array}$ & $\begin{array}{c}\text { HC vs RRMS } \\
\left(R_{E_{E M}}\right)\end{array}$ & $\begin{array}{l}\text { HC vs } \\
\text { PPMS }\end{array}$ \\
\hline Age (yr) & $48.0(7.3)$ & $46.9(7.0)$ & $48.6(7.0)$ & $47.4(6.8)$ & $.72(.72)$ & $.72(.72)$ & .72 \\
\hline Disease duration (yr) & - & $12.4(10.7)$ & $13.2(11.0)$ & $7.7(3.3)$ & $.006^{c}(.006)^{c}$ & - & - \\
\hline EDSS $^{d}$ & - & $2.5(2.5)$ & $4(0.6)$ & $4.5(1.6)$ & $<.001^{c}(.173)$ & - & - \\
\hline TILL $^{d}$ & - & $1.1(1.1)$ & $1.4(1.6)$ & $1.8(3.3)$ & $.023^{c}(.276)$ & - & - \\
\hline $\mathrm{T}^{2 \mathrm{LL}^{\mathrm{d}}}$ & - & $2.1(4.9)$ & $3.6(7.2)$ & $2.8(10.6)$ & $.431(.923)$ & - & - \\
\hline BPF (\%) & $86.2(1.5)$ & $84.2(2.4)$ & $83.8(2.4)$ & $84.7(3.0)$ & $.490(.490)$ & $<.001(<.001)$ & .08 \\
\hline $\operatorname{CCV}\left(\mathrm{cm}^{3}\right)$ & $4.6(0.6)$ & $4.1(0.7)$ & $4.0(0.7)$ & $4.1(0.8)$ & $.690(.690)$ & $.004(.001)$ & .013 \\
\hline
\end{tabular}

Note:- - indicates not relevant.

${ }^{a}$ The number of participants and the female/male ratio are reported for each group. Unless otherwise indicated, data are reported as mean (SD). Numbers in parentheses in the headers of columns $2-5$ are No. of subjects in groups (F/M).

${ }^{b}$ Pair-wise comparison ( $P$ value). Differences among HC, PPMS, and RRMS (RR $E_{E M}$ ) groups were tested using a $t$ test or Mann-Whitney $U$ test with nonpooled SD (BenjaminiHochberg correction).

c Significant.

${ }^{\mathrm{d}}$ Mann-Whitney $U$ test with nonpooled SD (Benjamini-Hochberg correction) (median and interquartile range).
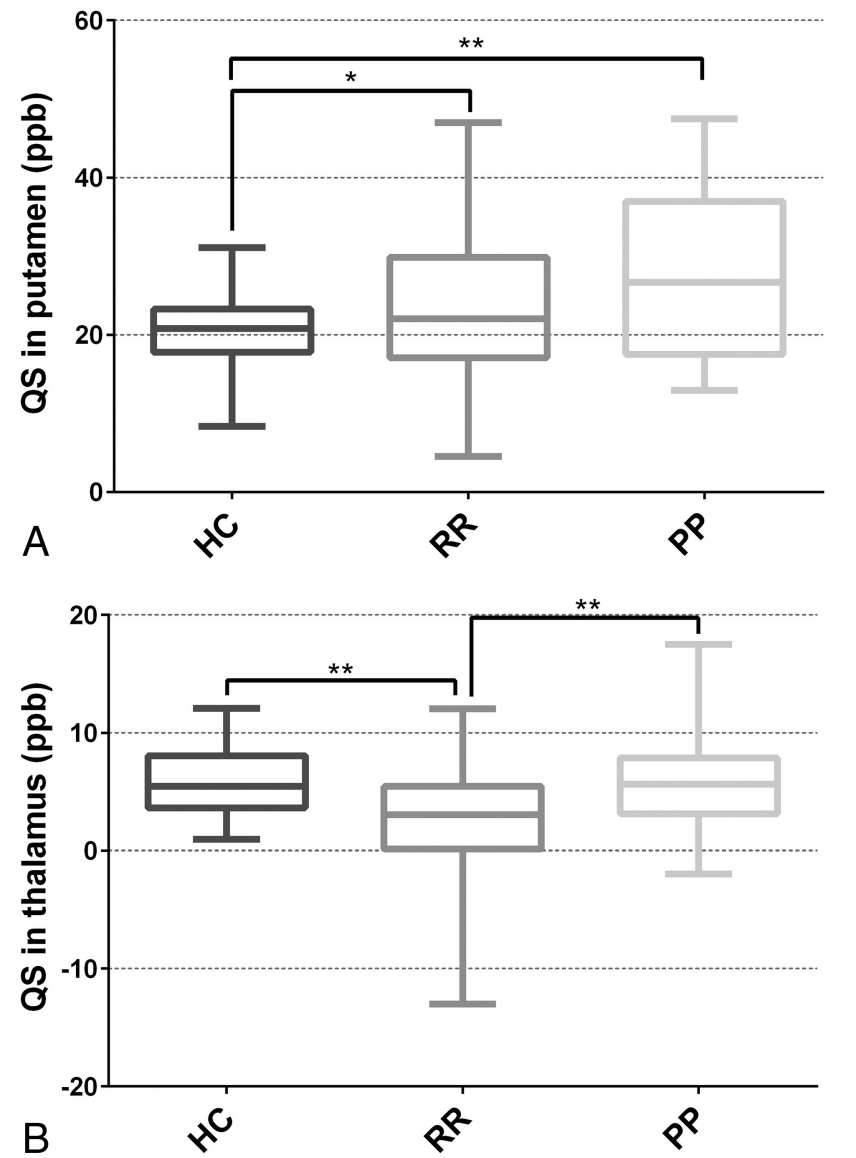

FIG 2. Quantitative susceptibility values in the putamen $(A)$ and thalamus $(B)$ across healthy controls and multiple sclerosis subtypes. Lower thalamic susceptibility values are observed in the RRMS compared with PPMS and HC groups. ppb indicates parts per billion.

because a single thalamic lesion was present in only 9 patients with RRMS (11\%) and 3 with PPMS (12.5\%).

Results from manual segmentation of the entire thalamus confirmed these findings, showing significantly lower QS values in RRMS compared with PPMS $(P=.026)$ and a trend toward lower values in RRMS compared with HC $(P=.057)$. When we analyzed thalamic substructures, the difference in QS among RRMS, PPMS, and HC groups was most pronounced in the thalamus without the pulvinar; in this region lower QS values were found in
RRMS compared with HC $(P=.035)$ and PPMS $(P=.035)$ groups. No significant differences were found in the pulvinar (RRMS versus $\mathrm{HC}, P=.28$; RRMS versus PPMS, $P=.28$ ) (Online Table 2 and On-line Fig 1).

\section{Correlations of QS Values with Conventional MR Imaging Metrics}

In the RRMS group, we identified negative correlations between QS values in the thalamus and T2LL as well as T1LL and a positive correlation with BPF and CCV. In the PPMS group, QS values did not correlate with any conventional MR imaging parameter (Table 3).

Interaction analysis between each combination of dependent (QS in CN, GP, Put, thalamus) and independent (T1LL, T2LL, $\mathrm{BPF}, \mathrm{CCV}$ ) variables in PPMS and RRMS confirmed significant interaction between MS subtypes and the T1LL/T2LL effect on thalamic QS $(P<.05)$. In the RRMS group, a strong negative linear relationship between T1LL/T2LL and QS in the thalamus was detected, while no such relationship was observed in the PPMS group (On-line Table 3 and On-line Fig 2).

\section{Association between MR Imaging Metrics and Disability (EDSS) in MS Subtypes}

In RRMS, the EDSS score was associated with all conventional MR imaging parameters: T1LL, T2LL, BPF, and CCV (Table 4). The strongest association was found for T1LL (Spearman correlation coefficient $=0.458, P<.0001)$. In addition, we identified a positive association between EDSS and QS values in the putamen (Fig 3A) and the CN. A negative association between the EDSS score and thalamic QS values was observed in the RRMS group (Fig 3C). In contrast, no association was found between the EDSS score and conventional MR imaging parameters in the PPMS group; there was only a positive association between EDSS and putaminal QS values observed in this group (Fig $3 B,-D$ ).

Interaction analysis comparing patients with EDSS $\leq 4$ and EDSS $>4$ in each MS subtype confirmed significant differences in mean thalamic susceptibility between RRMS with greater EDSS scores and PPMS regardless of EDSS scores $(P<.001)$ (On-line Tables 4 and 5). Statistical differences in T1LL and T2LL were observed only between RRMS with greater EDSS scores and 
Table 2: Quantitative susceptibility within deep GM structures ${ }^{a}$

\begin{tabular}{|c|c|c|c|c|c|c|c|}
\hline \multirow{2}{*}{$\begin{array}{c}\text { DGM Structure } \\
\text { QS (ppb) }\end{array}$} & \multirow[b]{2}{*}{$\mathrm{HC}$} & \multirow[b]{2}{*}{ RRMS } & \multirow[b]{2}{*}{$R_{E M} M S$} & \multirow[b]{2}{*}{ PPMS } & \multicolumn{3}{|c|}{ Pair-wise Comparison (P Value) ${ }^{b}$} \\
\hline & & & & & PPMS vs RRMS (RR EM $)$ & HC vs RRMS (RR EM) & HC vs PPMS \\
\hline $\mathrm{CN}$ & $32.6(9.5)$ & $35.0(9.6)$ & 38.3 (10.1) & $36.8(6.8)$ & $.390(.470)$ & $.390(.230)$ & .230 \\
\hline GP & $66.7(8.5)$ & $71.9(14.0)$ & 72.4 (14.1) & $70.8(13.0)$ & $.860(.860)$ & $.170(.170)$ & .420 \\
\hline Put & $20.3(5.4)$ & $24.3(10.7)$ & $27.7(10.3)$ & $28.1(10.5)$ & $.158(.899)$ & $.042^{c}(.003)^{c}$ & $.009^{c}$ \\
\hline Thal & $5.9(3.3)$ & $2.6(4.9)$ & $2.0(5.7)$ & $5.6(3.9)$ & $.007^{c}(.007)^{c}$ & $.004^{c}(.004)^{c}$ & .757 \\
\hline
\end{tabular}

Note:-Thal indicates thalamus

${ }^{a}$ All values are means (SD).

${ }^{b}$ Pair-wise comparisons ( $P$ value) were tested using $t$ tests with nonpooled SD (Benjamini-Hochberg correction).

c Significant.

Table 3: Correlations of QS values in DGM with conventional MRI parameters in the RRMS and PPMS groups

\begin{tabular}{|c|c|c|c|c|c|c|c|c|}
\hline \multirow[b]{2}{*}{ DGM Region } & \multicolumn{2}{|c|}{ TILL } & \multicolumn{2}{|c|}{ T2LL } & \multicolumn{2}{|c|}{ BPF } & \multicolumn{2}{|c|}{ CCV } \\
\hline & $r_{\mathrm{s}}$ & $P$ Value & $r_{\mathrm{s}}$ & $P$ Value & $r_{\mathrm{s}}$ & $P$ Value & $r_{\mathrm{s}}$ & $P$ Value \\
\hline \multicolumn{9}{|l|}{ RRMS } \\
\hline $\mathrm{CN}$ & -0.09 & .44 & -0.07 & .56 & -0.01 & .96 & -0.05 & .63 \\
\hline GP & 0.02 & .85 & 0.07 & .53 & -0.00 & .99 & -0.10 & .37 \\
\hline Put & 0.14 & .23 & 0.12 & .30 & -0.20 & .08 & -0.21 & .06 \\
\hline Thal & -0.36 & $.001^{\mathrm{a}}$ & -0.35 & $.001^{\mathrm{a}}$ & 0.23 & $.04^{\mathrm{a}}$ & 0.25 & $.02^{\mathrm{a}}$ \\
\hline \multicolumn{9}{|l|}{ PPMS } \\
\hline $\mathrm{CN}$ & -0.13 & .55 & -0.16 & .46 & 0.05 & .80 & 0.02 & .93 \\
\hline GP & 0.07 & .75 & 0.09 & .69 & -0.03 & .86 & 0.06 & .79 \\
\hline Put & 0.25 & .25 & 0.36 & .09 & -0.28 & .18 & -0.16 & .47 \\
\hline Thal & -0.04 & .85 & 0.03 & .90 & 0.14 & .52 & 0.11 & .60 \\
\hline
\end{tabular}

Note:- $r_{\mathrm{s}}$ indicates Spearman correlation coefficient; Thal, thalamus.

a Significant.

Table 4: Associations of EDSS with conventional MRI metrics and QS values

\begin{tabular}{|c|c|c|c|c|}
\hline & \multicolumn{2}{|c|}{ RRMS } & \multicolumn{2}{|c|}{ PPMS } \\
\hline & $r_{s}$ & $P$ Value & $r_{s}$ & $P$ Value \\
\hline \multicolumn{5}{|c|}{$\begin{array}{c}\text { Conventional MRI } \\
\text { metrics }\end{array}$} \\
\hline TILL & 0.458 & $<.001^{\mathrm{a}}$ & 0.195 & .361 \\
\hline T2LL & 0.336 & $.002^{\mathrm{a}}$ & 0.237 & .265 \\
\hline BPF & -0.281 & $.012^{a}$ & -0.001 & .995 \\
\hline $\mathrm{CCV}$ & -0.267 & $.017^{a}$ & -0.089 & .680 \\
\hline \multicolumn{5}{|l|}{ QS } \\
\hline $\mathrm{CN}$ & 0.234 & $.037^{a}$ & 0.127 & .554 \\
\hline GP & -0.106 & .350 & -0.059 & .785 \\
\hline Put & 0.298 & $.007^{a}$ & 0.464 & $.022^{\mathrm{a}}$ \\
\hline Thal & -0.251 & $.024^{\mathrm{a}}$ & 0.119 & .581 \\
\hline
\end{tabular}

Note:-Thal indicates thalamus.

a Significant.

RRMS with lower EDSS scores $(P=.004, P=.027$, respectively) (On-line Tables 4, 6, and 7).

\section{DISCUSSION}

Comparing iron concentration in DGM between PPMS and RRMS subtypes, we identified QS values in the thalamus as the only significant difference between age- and sex-matched RRMS and PPMS groups. EDSS was associated with conventional MR imaging parameters, indicating disease severity in the RRMS group. Additionally, the EDSS score was positively associated with iron concentration in the putamen and $\mathrm{CN}$ and negatively associated with thalamic iron. No associations between EDSS and conventional MR imaging metrics were found in the PPMS group.

Previous MR imaging studies detected similarities but also several differences between PPMS and RRMS. Lesion morphology (iron deposition, the presence of the central vein), lesion count, and the proportion of cortical-to-total lesion counts were similar in PPMS and RRMS groups. ${ }^{27}$ In PPMS, more diffuse abnormalities in the brain and spinal cord along with smaller caudate volume were apparent compared with RRMS. ${ }^{28}$ In PPMS, clinicoradiologic correlation was weak for cerebral T1LL/ T2LL but was stronger between spinal cord symptoms and spinal cord MR imaging parameters. ${ }^{29,30}$

In MS, increased iron levels seem to be already present in the clinically isolated syndrome group, and further buildup can be observed in the early stage after the transition to RRMS. ${ }^{16,31,32} \mathrm{We}$ confirmed significantly increased iron content in the putamen in both MS subtypes compared with $\mathrm{HC}$, in agreement with previous studies. ${ }^{4,6,7,33-35}$ While iron concentration was increased within the basal ganglia in patients with RRMS, the opposite (ie, lower iron concentration) was found in the thalamus. This result is consistent with findings in several previous MR R2* relaxometry studies showing lower thalamic iron in MS compared with HC. ${ }^{6,7}$ On the contrary, other MR imaging studies with R2*, QSM, magnetic field correlation imaging, or phase imaging showed no significant difference ${ }^{1,36}$ or even higher iron concentration in the thalamus in patients with RRMS. ${ }^{2,8,37,38}$

The cause of these contradictory findings is not clear. Because the disease duration of the RRMS group included in our study was rather long compared with other studies and thalamic iron content was negatively correlated with EDSS, one can speculate that iron may accumulate in the early MS stage, while its concentration may decrease in the later stage of the disease. However, another study indicated that the loss of thalamic iron in MS has already begun in patients with clinically isolated syndrome with further decrease after the conversion to definite MS. ${ }^{11}$ In normal aging, total thalamic iron shows a very different pattern from that in all other DGM structures, with an accumulation until the fourth decade, followed by a mild decrease. ${ }^{26}$ A histochemical study has shown that iron distribution in the thalamus is uneven, with the 


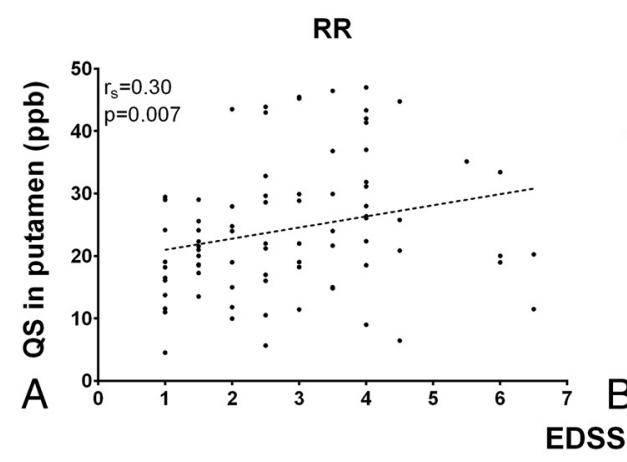

RR

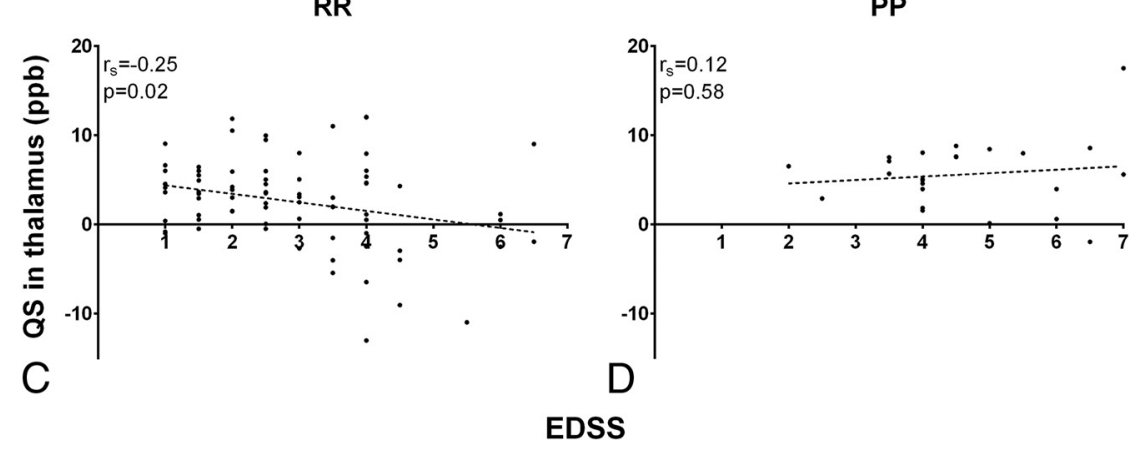

FIG 3. The relationship between clinical disability and quantitative susceptibility values in the putamen and thalamus across PPMS and RRMS groups. X-axes indicate EDSS, while y-axes indicate quantitative susceptibility values in parts per billion. A positive correlation between EDSS and putaminal quantitative susceptibility values is present in both the RRMS (A) and PPMS (B) groups. In the thalamus, a negative correlation between EDSS and quantitative susceptibility is present only in the RRMS group (C), while no correlation is observed in the PPMS group (D).

anterior nuclear group, dorsomedial group, and pulvinar having the highest iron reactivity. ${ }^{39}$ It is thus possible that the temporal pattern of iron concentration change in individual thalamic nuclei is different. Indeed, a cross-sectional MR imaging study with $\mathrm{R} 2^{*}$ and QSM showed that iron in the thalamic pulvinar may increase with aging, while there was no age-related change in other thalamic nuclei. ${ }^{40}$ In our study, the difference in thalamic QS values between RRMS on one hand and PPMS and HC on the other hand was mostly driven by variance in thalamic nuclei beyond the pulvinar, which is suggestive of diffuse iron loss within the thalamus.

Thalamic QS values in the PPMS group were significantly higher compared with the RRMS group; this finding indicates different regulation of thalamic iron concentration in these MS subtypes. However, the causes and consequences of different thalamic iron levels in RRMS and PPMS cannot be clarified from this cross-sectional study design. QS values in the thalamus were negatively correlated with T2LL and T1LL and with EDSS in the RRMS groups; this finding suggests an association between decreasing thalamic iron concentration and increasing WM impairment. This association between thalamic susceptibility and EDSS was not observed in the PPMS group. Susceptibility decrease may be due to lower (paramagnetic) iron or higher (diamagnetic) myelin content. ${ }^{14,41}$ Higher myelin content is not plausible in the context of MS; thus, our finding of negative thalamic susceptibility values in patients with RRMS and higher EDSS scores is rather consistent with the loss of iron content. Moreover, the T2LL in the thalamus, which could potentially affect susceptibility, was very low, and the difference in lesion count was insignificant in the RRMS and PPMS groups.

When we investigated the conventional MR imaging metrics, T1LL was higher in the PPMS compared with the RRMS group. This T1LL difference could be related to higher clinical severity in the PPMS group, and it was not observed when patients with PPMS and RRMS were matched for EDSS scores. T1 hypointense lesions often represent the final destructive tissue changes, and T1LL may better correlate with disease progression and disability. ${ }^{42}$ Di Perri et $\mathrm{al}^{43}$ have shown that T1LL was similar in PPMS and RRMS subtypes matched for disease duration. However, the T1LLto-T2LL ratio, along with clinical disability, was significantly higher in PPMS compared with RRMS. In other studies, on the contrary, the incidence of all brain lesion types has been reported to be reduced and brain atrophy increased in patients with PPMS compared with other MS subtypes, though there is a wide variability. ${ }^{12,29,44-46}$ The low number of patients with PPMS in our study and in other studies precludes firm conclusions, and further studies with larger PPMS cohorts are needed.

Examining associations between clinical severity and MR imaging parameters in the PPMS group, we only found a positive association between EDSS and iron concentration in the putamen. The correlation between iron concentration in the putamen and CN and clinical severity is well-established in MS, ${ }^{47,48}$ and it appears to be invariable regardless of the clinical subtype. Ropele et $\mathrm{al}^{4}$ included 7 patients with PPMS and 7 with secondary-progressive MS, in addition to 83 with RRMS in their cohort. They identified the EDSS score as an independent predictor of iron accumulation in the DGM. However, the results were likely driven by RRMS, while there were a limited number of patients with PPMS and SPMS. ${ }^{4}$ No significant association between conventional MR imaging metrics and EDSS has been detected in PPMS, which is consistent with findings in previous studies. ${ }^{43,49}$ In RRMS, there was a correlation between EDSS and conventional MR imaging parameters, which reflects WM lesions and brain atrophy. The correlation of EDSS with T1LL was stronger than with T2LL. These findings are in the line with previous studies ${ }^{23,42,50-55}$ showing that T1LL and T2LL provide a useful marker for disease progression and long-term therapeutic effect in RRMS.

To our best knowledge, this is the first study comparing iron concentration in DGM and its correlations with EDSS score between PPMS and RRMS subtypes. However, there are some limitations. Our PPMS group was rather small, and no MR imaging data were collected from the spinal cord, which could also contribute to the explanation of clinical disability. Cross-sectional 
design provides no information regarding the temporal dynamics of iron accumulation. Future work using a larger PPMS cohort in a longitudinal setting is needed. Likewise, spinal cord pathology, iron deposition within WM, and demyelinating lesions should be the focus of further investigations.

\section{CONCLUSIONS}

Our findings support the concept that PPMS is a part of the MS disease spectrum, not a separate entity. When patients with RRMS are matched to those with PPMS for age, sex, and EDSS, these groups do not differ regarding conventional MR imaging metrics. Decreased susceptibility within the thalamus was the only significant MR imaging difference between PPMS and RRMS groups, suggesting different thalamic iron metabolism according to the underlying MS subtype.

On the other hand, there are significant differences in the extent of correlation of MR imaging parameters and clinical severity between PPMS and RRMS subtypes. In RRMS, EDSS is significantly associated with conventional parameters and, additionally, with iron concentration in the striatum. In contrast, no association was found in PPMS, except a weak correlation with putaminal iron. These findings suggest that different driving forces of disability take effect in RRMS and PPMS subtypes.

Disclosures: Andrea Burgetova—RELATED: Grant: RVO VFN64165 (Czech Ministry of Health), Comments: Money went to the institution and a part of it to me*. Petr Dusek-RELATED: Grant: Czech Ministry of Health, 15-25602A. Manuela Vaneckova-RELATED: Grant: Czech Ministries of Health, RVO-VFN64165*; UNRELATED: Consultancy: Biogen Idec, Novartis, Merck Serono, Teva Pharmaceutical Industries; Payment for Lectures Including Service on Speakers Bureaus: Biogen Idec, Novartis, Merck Serono, Teva Pharmaceutical Industries; Travel/Accommodations/ Meeting Expenses Unrelated to Activities Listed: Biogen Idec, Genzyme. Dana Horakova-UNRELATED: Consultancy: Biogen Idec, Sanofi-Genzyme; Grants/Grants Pending: grants GA CR 16-03322S and PRVOUK P26/LF/4*; Payment for Lectures Including Service on Speakers Bureaus: Biogen Idec, Novartis, Merck, Bayer HealthCare Pharmaceuticals, Sanofi-Genzyme, and Teva Pharmaceutical Industries. Jan Krasensky—RELATED: Grant: Ministry of Health, RVO-VFN-64165*; UNRELATED: Travel/Accommodations/Meeting Expenses Unrelated to Activities Listed: Biogen Idec, Novartis. Lukas Sobisek—RELATED: Grant: Czech Ministry of Education project PRVOUK-P26/LF1/4; Other: Novartis; UNRELATED: Employment: University of Economics in Prague, Comments: assistant in Department of Statistics and Informatics. Zdenek Seidl-RELATED: Grant: Ministry of Health, RVO-VFN64165.* *Money paid to the institution.

\section{REFERENCES}

1. Raz E, Branson B, Jensen JH, et al. Relationship between iron accumulation and white matter injury in multiple sclerosis: a case-control study. J Neurol 2015;262:402-09 CrossRef Medline

2. Cobzas D, Sun H, Walsh AJ, et al. Subcortical gray matter segmentation and voxel-based analysis using transverse relaxation and quantitative susceptibility mapping with application to multiple sclerosis. J Magn Reson Imaging 2015;42:1601-10 CrossRef Medline

3. Stankiewicz JM, Neema M, Ceccarelli A. Iron and multiple sclerosis. Neurobiol Aging 2014;35(suppl 2):S51-58 CrossRef Medline

4. Ropele S, Kilsdonk ID, Wattjes MP, et al. Determinants of iron accumulation in deep grey matter of multiple sclerosis patients. Mult Scler 2014;20:1692-98 CrossRef Medline

5. Ropele S, de Graaf W, Khalil M, et al. MRI assessment of iron deposition in multiple sclerosis. J Magn Reson Imaging 2011;34:13-21 CrossRef Medline

6. Khalil M, Langkammer C, Ropele S, et al. Determinants of brain iron in multiple sclerosis: a quantitative 3T MRI study. Neurology 2011; 77:1691-97 CrossRef Medline

7. Burgetova A, Seidl Z, Krasensky J, et al. Multiple sclerosis and the accumulation of iron in the basal ganglia: quantitative assessment of brain iron using MRI t(2) relaxometry. Eur Neurol 2010;63: 136-43 CrossRef Medline

8. Ge Y, Jensen JH, Lu H, et al. Quantitative assessment of iron accumulation in the deep gray matter of multiple sclerosis by magnetic field correlation imaging. AJNR Am J Neuroradiol 2007;28:1639-44 CrossRef Medline

9. Haider L, Simeonidou C, Steinberger G, et al. Multiple sclerosis deep grey matter: the relation between demyelination, neurodegeneration, inflammation and iron. J Neurol Neurosurg Psychiatry 2014;85: 1386-95 CrossRef Medline

10. Hametner S, Wimmer I, Haider L, et al. Iron and neurodegeneration in the multiple sclerosis brain. Ann Neurol 2013;74:848-61 CrossRef Medline

11. Khalil M, Langkammer C, Pichler A, et al. Dynamics of brain iron levels in multiple sclerosis: a longitudinal 3T MRI study. Neurology 2015;84:2396-402 CrossRef Medline

12. Antel J, Antel S, Caramanos Z, et al. Primary progressive multiple sclerosis: part of the MS disease spectrum or separate disease entity? Acta Neuropathol 2012;123:627-38 CrossRef Medline

13. Dusek $P$, Dezortova M, Wuerfel J. Imaging of iron. Int Rev Neurobiol 2013;110:195-239 CrossRef Medline

14. Langkammer C, Schweser F, Krebs N, et al. Quantitative susceptibility mapping (QSM) as a means to measure brain iron? A post mortem validation study. Neuroimage 2012;62:1593-99 CrossRef Medline

15. Schmalbrock P, Prakash RS, Schirda B, et al. Basal ganglia iron in patients with multiple sclerosis measured with 7T quantitative susceptibility mapping correlates with inhibitory control. AJNR Am J Neuroradiol 2016;37:439-46 CrossRef Medline

16. Al-Radaideh AM, Wharton SJ, Lim SY, et al. Increased iron accumulation occurs in the earliest stages of demyelinating disease: an ultra-high field susceptibility mapping study in clinically isolated syndrome. Mult Scler 2013;19:896-903 CrossRef Medline

17. Wang Y, Liu T. Quantitative susceptibility mapping (QSM): decoding MRI data for a tissue magnetic biomarker. Magn Reson Med 2015;73:82-101 CrossRef Medline

18. Schweser F, Deistung A, Lehr BW, et al. Differentiation between diamagnetic and paramagnetic cerebral lesions based on magnetic susceptibility mapping. Med Phys 2010;37:5165-78 CrossRef Medline

19. Lublin FD, Reingold SC, Cohen JA, et al. Defining the clinical course of multiple sclerosis: the 2013 revisions. Neurology 2014;83:278-86 CrossRef Medline

20. Ho DE, Imai K, King G, et al. MatchIt: nonparametric preprocessing for parametric causal inference. J Stat Soft 2011;42:1-28

21. Horakova D, Kalincik T, Dolezal O, et al. Early predictors of nonresponse to interferon in multiple sclerosis. Acta Neurol Scand 2012; 126:390-97 CrossRef Medline

22. Kalincik T, Vaneckova M, Tyblova M, et al. Volumetric MRI markers and predictors of disease activity in early multiple sclerosis: a longitudinal cohort study. PLoS One 2012;7:e50101 CrossRef Medline

23. Vaneckova M, Kalincik T, Krasensky J, et al. Corpus callosum atrophy: a simple predictor of multiple sclerosis progressiona longitudinal 9-year study. Eur Neurol 2012;68:23-27 CrossRef Medline

24. Langkammer C, Bredies K, Poser BA, et al. Fast quantitative susceptibility mapping using $3 \mathrm{D}$ EPI and total generalized variation. $\mathrm{Neu}$ roimage 2015;111:622-30 CrossRef Medline

25. Yushkevich PA, Piven J, Hazlett HC, et al. User-guided 3D active contour segmentation of anatomical structures: significantly improved efficiency and reliability. Neuroimage 2006;31:1116-28 CrossRef Medline

26. Hallgren B, Sourander P. The effect of age on the non-haemin iron in the human brain. J Neurochem 1958;3:41-51 CrossRef Medline

27. Kuchling J, Ramien C, Bozin I, et al. Identical lesion morphology in primary progressive and relapsing-remitting MS: an ultrahigh field MRI study. Mult Scler 2014;20:1866-71 CrossRef Medline

28. Jonkman LE, Rosenthal DM, Sormani MP, et al. Gray matter corre- 
lates of cognitive performance differ between relapsing-remitting and primary-progressive multiple sclerosis. PLoS One 2015;10: e0129380 CrossRef Medline

29. Galego O, Gouveia A, Batista S, et al. Brain atrophy and physical disability in primary progressive multiple sclerosis: a volumetric study. Neuroradiol J 2015;28:354-58 CrossRef Medline

30. Nijeholt GJ, van Walderveen MA, Castelijns JA, et al. Brain and spinal cord abnormalities in multiple sclerosis: correlation between MRI parameters, clinical subtypes and symptoms. Brain 1998; 121(pt 4):687-97 CrossRef Medline

31. Hagemeier J, Heininen-Brown M, Poloni GU, et al. Iron deposition in multiple sclerosis lesions measured by susceptibility-weighted imaging filtered phase: a case control study. J Magn Reson Imaging 2012;36:73-83 CrossRef Medline

32. Quinn MP, Gati JS, Klassen ML, et al. Increased deep gray matter iron is present in clinically isolated syndromes. Mult Scler Relat Disord 2014;3:194-202 CrossRef Medline

33. Hammond KE, Metcalf M, Carvajal $\mathrm{L}$, et al. Quantitative in vivo magnetic resonance imaging of multiple sclerosis at 7 Tesla with sensitivity to iron. Ann Neurol 2008;64:707-13 CrossRef Medline

34. Khalil M, Enzinger C, Langkammer C, et al. Quantitative assessment of brain iron by $\mathrm{R}(2)^{\star}$ relaxometry in patients with clinically isolated syndrome and relapsing-remitting multiple sclerosis. Mult Scler 2009;15:1048-54 CrossRef Medline

35. Zivadinov R, Heininen-Brown M, Schirda CV, et al. Abnormal subcortical deep-gray matter susceptibility-weighted imaging filtered phase measurements in patients with multiple sclerosis: a case-control study. Neuroimage 2012;59:331-39 CrossRef Medline

36. Du S, Sah SK, Zeng C, et al. Iron deposition in the gray matter in patients with relapse-remitting multiple sclerosis: a longitudinal study using three-dimensional (3D)-enhanced $\mathrm{T} 2{ }^{*}$-weighted angiography (ESWAN). Eur J Radiol 2015;84:1325-32 CrossRef Medline

37. Hagemeier J, Yeh EA, Brown MH, et al. Iron content of the pulvinar nucleus of the thalamus is increased in adolescent multiple sclerosis. Mult Scler 2013;19:567-76 CrossRef Medline

38. Modica CM, Zivadinov R, Dwyer MG, et al. Iron and volume in the deep gray matter: association with cognitive impairment in multiple sclerosis. AJNR Am J Neuroradiol 2015;36:57-62 CrossRef Medline

39. Morris CM, Candy JM, Oakley AE, et al. Histochemical distribution of non-haem iron in the human brain. Acta Anat (Basel) 1992;144: 235-57 CrossRef Medline

40. Acosta-Cabronero J, Betts MJ, Cardenas-Blanco A, et al. In vivo MRI mapping of brain iron deposition across the adult lifespan. J Neurosci 2016;36:364-74 CrossRef Medline

41. Fukunaga M, Li TQ, van Gelderen P, et al. Layer-specific variation of iron content in cerebral cortex as a source of MRI contrast. Proc Natl Acad Sci U S A 2010;107:3834-39 CrossRef Medline
42. Truyen L, van Waesberghe JH, van Walderveen MA, et al. Accumulation of hypointense lesions ("black holes") on T1 spin-echo MRI correlates with disease progression in multiple sclerosis. Neurology 1996;47:1469-76 CrossRef Medline

43. Di Perri C, Battaglini M, Stromillo ML, et al. Voxel-based assessment of differences in damage and distribution of white matter lesions between patients with primary progressive and relapsing-remitting multiple sclerosis. Arch Neurol 2008;65:236-43 Medline

44. Miller DH, Leary SM. Primary-progressive multiple sclerosis. Lancet Neurol 2007;6:903-12 CrossRef Medline

45. Stevenson VL, Miller DH, Rovaris M, et al. Primary and transitional progressive MS: a clinical and MRI cross-sectional study. Neurology 1999;52:839-45 CrossRef Medline

46. Thompson AJ, Montalban X, Barkhof F, et al. Diagnostic criteria for primary progressive multiple sclerosis: a position paper. $\mathrm{Ann} \mathrm{Neu}$ rol 2000;47:831-35 Medline

47. Zhang Y, Metz LM, Yong VW, et al. 3T deep gray matter T2 hypointensity correlates with disability over time in stable relapsing-remitting multiple sclerosis: a 3-year pilot study. J Neurol Sci 2010; 297:76-81 CrossRef Medline

48. Tjoa CW, Benedict RH, Weinstock-Guttman B, et al. MRI T2 hypointensity of the dentate nucleus is related to ambulatory impairment in multiple sclerosis. J Neurol Sci 2005;234:17-24 CrossRef Medline

49. Ruggieri S, Petracca M, Miller A, et al. Association of deep gray matter damage with cortical and spinal cord degeneration in primary progressive multiple sclerosis. JAMA Neurol 2015;72:1466-74 CrossRef Medline

50. Bergsland N, Horakova D, Dwyer MG, et al. Subcortical and cortical gray matter atrophy in a large sample of patients with clinically isolated syndrome and early relapsing-remitting multiple sclerosis. AJNR Am J Neuroradiol 2012;33:1573-78 CrossRef Medline

51. Bermel RA, Bakshi R. The measurement and clinical relevance of brain atrophy in multiple sclerosis. Lancet Neurol 2006;5:158-70 CrossRef Medline

52. Bodini B, Battaglini M, De Stefano N, et al. T2 lesion location really matters: a 10-year follow-up study in primary progressive multiple sclerosis. J Neurol Neurosurg Psychiatry 2011;82:72-77 CrossRef Medline

53. Ciccarelli O, Brex PA, Thompson AJ, et al. Disability and lesion load in MS: a reassessment with MS functional composite score and 3D fast FLAIR. J Neurol 2002;249:18-24 CrossRef Medline

54. Kearney H, Rocca MA, Valsasina P, et al. Magnetic resonance imaging correlates of physical disability in relapse onset multiple sclerosis of long disease duration. Mult Scler 2014;20:72-80 CrossRef Medline

55. Zivadinov R, Stosic M, Cox JL, et al. The place of conventional MRI and newly emerging MRI techniques in monitoring different aspects of treatment outcome. J Neurol 2008;255(suppl 1):61-74 CrossRef Medline 